\title{
The partial response SVM
}

\author{
B. Walters, S. Ortega-Martorell, I. Olier and P.J.G. Lisboa*
}

Data Science Research Centre, School of Computer Science and Mathematics Liverpool John Moores University, Byrom Street, Liverpool L3 3AF, UK

\begin{abstract}
We introduce a probabilistic algorithm for binary classification based on the SVM through the application of the ANOVA decomposition for multivariate functions to express the logit of the Platt estimate of the posterior probability as a non-redundant sum of functions of fewer variables (partial responses) followed by feature selection with the Lasso. The partial response SVM (prSVM) is compared with previous interpretable models of the SVM. Its accuracy and stability are demonstrated with real-world data sets.
\end{abstract}

\section{Introduction}

\subsection{Motivation}

Black-box models are not interpretable by design, lacking transparency and accountability [1]. In particular for binary classification the SVM is a powerful discriminant model, but its deployment in high-stakes applications is limited by two factors: first, its roots in computational learning theory do not conform to the models of chance variation that are required to infer a posterior probability; second, the use of Gaussian makes it difficult to quantify the exact weight of each input towards the model output. As a consequence, the end user does not know for instance why specifically an SVM has misclassified for a particular example [2]. The first aspect has been the subject of extended research. Arguably the simplest approach to take is to use Platt's approximation [3] by re-calibrating the decision function of the SVM i.e. the risk score function, by using a logistic regression model with parameters estimated by minimising the standard negative log-likelihood. We use this approximation but make the logistic regression model multivariate by applying it to multiple components derived from the decision function.

Explanations for model decisions, however, can be pivotal in users making decisions e.g. in medical decision support [4]. There are several definitions of transparency and interpretability throughout the literature, but five main desiderata have been proposed for robust interpretability and explainability [5,6], that will guide the proposed approach: Intelligibility: "Are the explanations immediate and understandable?"; Faithfulness: "Are relevance scores indicative of 'true' importance?"; Stability: "How consistent are the explanations for neighbouring examples?"; Parsimony: "Do the explanatory variables comprise a minimal set?"; and Consistency: "How robust are the explanations to perturbations in the data?". In our view, an interpretable method that can fit these five criteria is the key to opening the black box corresponding to a standard $l_{l}$-regularised SVM.

\footnotetext{
* Bradley Walters would like to thank Liverpool John Moores University for a PhD Studentship
} 
A key aim of the proposed approach is to create a method that focuses on contribution rather than attribution. In this context we refer to attribution as a signal fed back from the model output to the input for the purposes of quantifying the sensitivity of the former to the latter. This may be derived from a local tangent model, such as LIME [4] providing an explanation at a specific data point about which variables are most important. In contrast, we refer to interpretability as the calculation feedforward of the exact contribution of each input to the model output, similar to what is the case in Generalised Additive Models (GAM). In particular, we aim to provide global interpretability over the whole data space by quantifying how much each variable contributes to a prediction.

\subsection{Related work}

Early approaches to interpreting SVMs sought to express them with Boolean rules and by identifying prototypes through clustering [7]. Later, it was suggested to use GAMs to interpret different machine learning models [8]. This built on a very early proposal to structure the neural networks as self-explaining models [9] that cross-over between machine learning and conventional statistics. However, the central issue of the selection of relevance features in a statistically principled way. This is critical to the efficient estimation of an interpretable model, especially when two-way effects are considered.

The proposed approach is closely related to the SVM nomogram [10,11] which followed a similar argument also with Platt's approximation, by considering a Taylor expansion of the Gaussian kernels, followed by an iterative re-weighted SVM applied to the component functions generated by the Taylor expansion, in order to reduce the number of required functions and provide stability to the model.

In fact, the expansion used in Van Belle's papers [10,11] and in this manuscript to express the multivariate Gaussian kernel as a sum of functions of fewer variables has the form of an ANOVA decomposition, which is finite and exact if all terms are taken into account. That is to say, for a $p$-dimensional input, it is composed of $2^{p}$ terms comprising a constant and effects of order $1,2, \ldots, p$. The assumption made in both papers is that in many real-world applications the signal-to-noise level will render higher-order terms less relevant because they are very difficult to infer accurately.

Therefore, there is potential for, and possibly even a performance advantage to be gained in making the decomposition of the complex multivariate kernel into simpler functions of fewer variables, which we call partial responses. This way, the coefficients of the partial functions, which we model for univariate and bivariate effects, can be explicitly estimated and further, by truncating the ANOVA expansion, the signal-to-noise ratio of the risk score functions can be improved by removing the higher-order, noisier terms, that are implicit in the original multivariate function.

The SVM nomogram followed earlier work [12] in the framework of Functional Data Analysis (FDA). This involves setting specialised regularisation terms to implement sparsity and smoothness in the risk score function, by driving down its derivative in specific ways. This makes the method of particular interest to certain types of data with correlated inputs e.g. smooth spectra and time series. 


\subsection{Novel contribution and limitations of this work}

The main contribution of this paper is an alternative methodology to calculate a nomogram for the SVM, by replacing the second SVM iteration in the model of [11] with the application of the logistic regression Lasso [13]. This has the advantage over the iterative re-weighted step of involving probabilistic modelling at the level of the partial responses including feature selection. This potentially improves calibration and stability in the selection of the final sparse model. It also simplifies the implementation of the SVM nomogram, so making it more easily accessible to other researchers.

The main limitation of this work is that it applies only to tabular data, comprising independent covariates, as distinct from structured data such as images, speech and text.

\section{Methodology}

The implementation of the partial response SVM (prSVM) is straightforward:

i. Calculate the ANOVA decomposition to any desired order anchored at a suitable point - we choose to use the median of data;

ii. Take the component functions, which are the partial responses at the anchor point, to be the covariates in logistic regression Lasso.

The first step is identical to the calculation of component functions in $[10,11]$. The difference in this paper is the application of a probabilistic method directly to the partial responses, rather than to the score function arising from a linear SVM as the second step. This step involves aggressive pruning of the model coefficients, which required an iterative re-weighted algorithm where the regularisation parameters of the linear SVM were inversely proportional to the size of the corresponding model coefficients. Hence, the smaller the coefficient, the faster it would be pushed towards zero. Effective as this approach proved to be, it can be less stable than the Lasso. In addition, the availability of many coefficients for calibration, compared with a scalar risk score, makes it easier to achieve good calibration.

It can be said that the prSVM utilises the benefits of the two models in a complementary way: the SVM contributes discriminant functions and the logistic Lasso carries out efficient feature selection.

More formally, the partial responses are obtained from the logit of the Platt approximation to the probability of class membership, in other words directly from the risk score of the SVM, by evaluating it at the median of the data, then allowing one variable to change at a time, then two. The key is to formulate an orthogonal decomposition so that, for $p$-dimensional input data, the terms added up to interactions of order $p$ exactly match the original function. The intention is to truncate this decomposition at order 2 as it is empirically observed that for many real-world applications e.g. in medicine, higher-order interactions seldom play a part in risk models, not least as low signal-to-noise ratios will make it difficult to infer such interactions accurately with reasonable sample sizes.

The ANOVA decomposition anchored at the origin is defined by: 


$$
\operatorname{logit}(P(C \mid x)) \approx \varphi(0)+\sum_{i} \varphi_{i}\left(x_{i}\right)+\sum_{i \neq j} \varphi_{i j}\left(x_{i}, x_{j}\right)+O\left(x_{i}, x_{j}, x_{i}\right)
$$

where:

$$
\begin{aligned}
& \varphi(0)=\operatorname{logit}(P(C \mid 0)) \\
& \varphi\left(x_{i}\right)=\operatorname{logit}\left(P\left(C \mid\left(0, \ldots, x_{i}, \ldots, 0\right)\right)\right)-\varphi(0) \\
& \varphi\left(x_{i}, x_{j}\right)=\operatorname{logit}\left(P\left(C \mid\left(0, \ldots, x_{i}, \ldots, x_{j}, \ldots, 0\right)\right)\right)-\varphi\left(x_{i}\right)-\varphi\left(x_{j}\right)-\varphi(0)
\end{aligned}
$$

Having standardised the covariates to unit variance and shifted the origin to the median, the values of the partial responses $\varphi$ above for each row of data, become the inputs to a standard logistic regression Lasso [13].

\section{Empirical evaluation}

\subsection{Data description}

The prSVM performance is compared with that of the original SVM with a Gaussian kernel and the SVM nomogram model [11] using the same two real-world data sets.

The Pima diabetes dataset $(n=532)$ comprises measurements from women aged over 21 years old, of Pima Indian heritage, tested for diabetes. There are 7 covariates and the binary outcome classes have a prevalence of $33.27 \%$.

The German Credit Card dataset ( $\mathrm{n}=1000)$ using the same 6 covariates as [11] for comparability and outcomes of good or bad credit risk with a prevalence of $30 \%$.

\subsection{Classification performance}

The two models for real-world data were optimised by 4-fold cross validation on the training data. For both models the hyperparameter $\sigma$ was tested in the range $\left[2^{-7}, 2^{2}\right]$ with the values $2^{-2}$ and $2^{-4}$ selected for the Pima and German Credit Card datasets respectively.

The relative performance compared to the original SVM and the values quoted in [11] are listed in Table 1. Note the smaller number of variables selected, for a similar classification performance. This is important because smaller univariate and bivariate effects are more difficult to infer accurately and can be unstable.

The implementation of SVM in R by [14] involves a cost parameter that penalises misclassifications. The effect on calibration of both the hyperparameters was considered and the calibration for the Pima dataset is shown in fig. 1. This is consistent with the hypothesis that modelling the SVM with component functions renders the prSVM a more accurate probabilistic model than resorting to the Platt approximation. The form of the partial responses provides valuable insights about the validity of the model predictions, as it can be verified by expert end-users. 
ESANN 2021 proceedings, European Symposium on Artificial Neural Networks, Computational Intelligence and Machine Learning. Online event, 6-8 October 2021, i6doc.com publ., ISBN 978287587082-7.

Available from http://www.i6doc.com/en/.

\begin{tabular}{|c|c|c|c|c|}
\hline Dataset & Model & AUC [CI] & $\begin{array}{c}\# \\
\text { components }\end{array}$ & $\begin{array}{c}\text { H-L statistic } \\
\text { (p-value) }\end{array}$ \\
\hline \multirow{2}{*}{$\begin{array}{c}\text { PIMA } \\
\text { Diabetes }\end{array}$} & SVM & $0.801[0.730,0.873]$ & 7 & $26.5(0.000867)$ \\
\cline { 2 - 5 } & prSVM & $0.806[0.737,0.876]$ & 7 & $15.7(0.0465)$ \\
\cline { 2 - 5 } & SVM & 0.780 & 28 & \\
\hline $\begin{array}{c}\text { German } \\
\text { Credit } \\
\text { Card }\end{array}$ & Sprox. [11] & & & \\
\cline { 2 - 5 } & prSVM & $0.757[0.696,0.818]$ & 6 & $21.0(0.00719)$ \\
\cline { 2 - 5 } & SVM & $0.696,0.813]$ & 18 & $11.2(0.190)$ \\
\hline
\end{tabular}

Table 1: Results comparison between the original SVM, the prSVM and the SVM

Approximation in [11]. H-L stands for Hosmer-Lemeshow test statistic. The \#components is the \#covariates for the SVM and the \#partial responses for the rest.
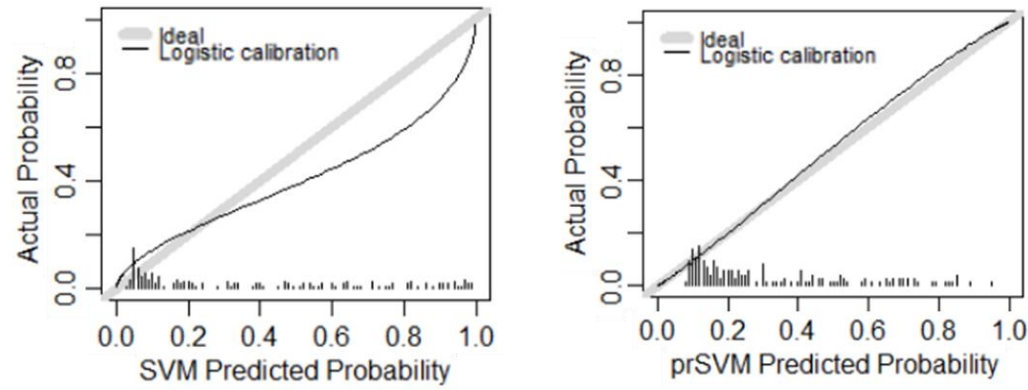

Fig. 1: Calibration curves for the Pima diabetes data set, with hyperparameters $\sigma=2^{-2}$ and Cost $=10^{-2}$, showing an improvement for the prSVM compared with the original SVM with a Gaussian kernel.
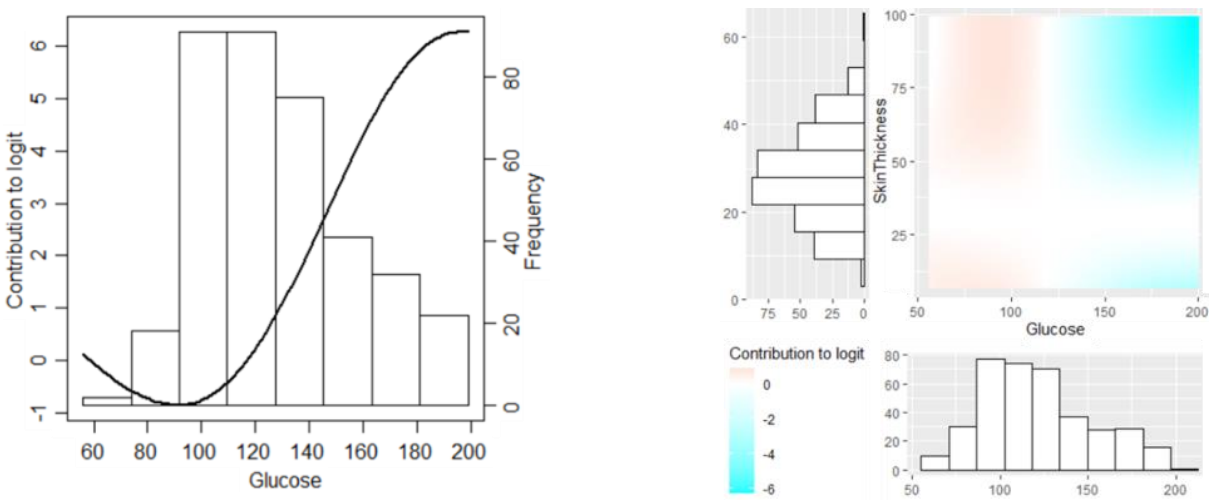

Fig. 2: Example partial responses in the nomogram for the Pima diabetes data set.

The contribution of each variable to the logit is shown on the y-axis. For a given observation, these contributions add to form the nomogram. The responses show the weights for Glucose and a 2-way interaction between Glucose and Skin thickness. 
ESANN 2021 proceedings, European Symposium on Artificial Neural Networks, Computational Intelligence and Machine Learning. Online event, 6-8 October 2021, i6doc.com publ., ISBN 978287587082-7.

Available from http://www.i6doc.com/en/.

\section{Conclusion}

An alternative proposal for the calculation of the SVM nomogram is presented. Compared with the original formulation in [11] the new approach is more stable to generate sparse models. It shows markedly better calibration than the original SVM while retaining a comparable classification performance.

A possible extension of this model is to calculate confidence intervals for the partial responses by re-sampling with the bootstrap. This is particularly important for quantifying uncertainty in underpopulated regions of the training data sample, shown in the histograms along the axes in fig. 2.

\section{References}

[1] C. Rudin, Stop explaining black box machine learning models for high stakes decisions and use interpretable models instead. Nature Machine Intelligence, 1:206-215, 2019.

[2] Y. Liang, S. Li, C. Yan, M. Li and C. Jiang, Explaining the black-box model: A survey of local interpretation methods for deep neural networks. Neurocomputing, 419:168-182, 2021.

[3] J.C. Platt, Probabilistic Outputs for Support Vector Machines and Comparisons to Regularized Likelihood Methods. In A.J. Smola, P. Bartlett, B. Schoelkopf, D. Schuurmans, editors, Advances in large margin classifiers. Cambridge, MA, USA: MIT Press, pages. 61-74, 1999.

[4] M.T. Ribeiro, S. Singh and C. Guestrin, "Why should I trust you?" Explaining the predictions of any classifier. In Proceedings of the 22nd ACM SIGKDD international conference on knowledge discovery and data mining, pages 1135-1144, 2016.

[5] D. Alvarez-Melis and T.S. Jaakkola, Towards robust interpretability with self-explaining neural networks. In Proceedings of the 32nd International Conference on Neural Information Processing Systems (NIPS'18), pages 7786-7795, 2018.

[6] P.J.G Lisboa, S. Ortega-Martorell, M. Jayabalan and I. Olier, Efficient Estimation of General Additive Neural Networks: A Case Study for CTG Data. In Joint European Conference on Machine Learning and Knowledge Discovery in Databases, Springer, Cham, pages 432-446, 2020.

[7] H. Núñez, C. Angulo, and A. Català, Rule extraction from support vector machines. In Proceedings of the European Symposium on Artificial Neural Networks (ESANN'02), pages 107-112, 2002.

[8] Y. Lou, R. Caruana, and J. Gehrke, Intelligible models for classification and regression. In Proceedings of the 18th ACM SIGKDD International Conference on Knowledge Discovery and Data Mining (KDD’12), ACM, pages 150-158, 2012.

[9] W.S. Sarle, Neural Networks and Statistical Models. In Proceedings of the Nineteenth Annual SAS Users Group International Conference, 1994.

[10] V. Van Belle and P.J.G. Lisboa, White box radial basis function classifiers with component selection for clinical prediction models. Artificial Intelligence in Medicine, 60:53-64, 2014.

[11] V. Van Belle, B. Van Calster, S. Van Huffel, J. Suykens and P. Lisboa, Explaining Support Vector Machines: A Color Based Nomogram. PLOS ONE, 11(10), 2016.

[12] B. Martin-Barragan, R. Lillo and J. Romo, Interpretable support vector machines for functional data. European Journal of Operational Research, 232:146-155, 2012.

[13] L. Meier, S. Van De Geer and P. Bühlmann, The group lasso for logistic regression. Journal of the Royal Statistical Society: Series B (Statistical Methodology), 70:53-71, 2008.

[14] A. Karatzoglou, D. Meyer, K. Hornik, Support Vector Machines in R. Journal of Statistical Software, 15:1-28, 2006. 